\begin{abstract}
Police officers around the world respond to and investigate calls regarding domestic violence (DV) daily. Police departments operate with standard protocols, particularly when engaging in investigations that involve allegations of strangulation or sexual assault. Operating under advisement of the Maricopa County Attorney’s Office, the Mesa Police Department (Mesa, AZ) has established protocols for detecting, recording, and prosecuting DV cases that involve strangulation and sexual assault. Allegations of strangulation (defined as impeded breathing) or sexual assault prompt officers to offer a sexual assault nursing exam (SANE) combined with strangulation treatment by forensic nurses at the Mesa Family Advocacy Center. Recognizing the potential for head injury to the assault victim in all DV situations, including intimate partner violence (IPV), the police department has added concussion-awareness training, as well as a point-of-incident investigative tool for its officers to record neurological function of the victim. Officers were instructed to use the ConQVerge device to measure and record the Near Point of Convergence (NPC) as a test of neurological impairment in suspected head injuries. In this article, we discuss the challenges and opportunities for assisting victims of DV strangulation and sexual assault (including non-DV sexual assault) with on-site assessment and consent for further medical assessment and treatment. Additionally, rates of domestic assault victims that report a crime, but decline to follow through with forensic medical tests, are reported for the first time. Lessons learned from the project that inform strategic operations in this space are offered to other agencies prior to the implementation of similar procedures.
\end{abstract}

Keywords: DV, intimate partner violence, traumatic brain injury, officers, assessment, strangulation, sexual assault 


\section{Involving police departments in early awareness of concussion \\ symptoms during domestic violence calls}

\section{Introduction}

In 2012 the Maricopa County Attorney’s Office (MCAO) implemented protocols to provide more effective medical care in instances where a domestic assault victim reported being strangled by an intimate partner. Strangulation is a predictor of homicide in domestic violence cases (Glass et al., 2008), as illustrated by $53 \%$ of 57 homicides in 1995-96 were preceded by one or more strangulation incidents (Block \& Devitt, 2000; Block \& Devitt, 2004). In a prospective review of their own cases, the San Diego City Attorney's Office reported a landmark study that outlined the value of objective forensic evidence, including photographic evidence, collected by medical providers, to support a strong prosecution (Hawley, McClane, \& Strack, 2001; McClane, Strack, \& Hawley, 2001; Strack, McClane, \& Hawley, 2001), which could reduce the incidence of subsequent homicide. The adoption of a countywide strangulation education and medical examination protocol resulted in significantly more felony arrests and convictions for DV cases with allegations of strangulation. The MCAO estimates that protocols for strangulation education and victim forensic examination increased DV strangulation cases filed for prosecution from $\sim 14 \%$ to $61.5 \%$ in a 2011 pilot program. Ongoing protocol implementation achieves a 55\% prosecution filing rate for DV strangulation.

In late 2016, the MCAO contacted the Mesa Police Department (MPD), a large municipal police agency, and a coalition of entities to formulate an approach to investigate incidences of traumatic brain injury (TBI; e.g. concussions) during domestic violence and sexual assault events, and to procure medical care for the victims. The group consisted of MPD, MCAO, victim's advocates/social workers, forensic nurse examiners (HonorHealth), a medical school 
translational neuroscience research team (University of Arizona College of Medicine - Phoenix), and a non-profit organization (The CACTIS Foundation). Over the course of a year, regular meetings were held to establish purpose, protocols, and procedures as to what role each organization would fill during the investigation of each case, and to report on progress and hurdles. Numerous legal issues ranging from the criminal investigations to medical treatment were discussed and navigated.

\section{Purpose}

This multi-faceted project sought to capture data about the incidence and consequences of TBI during DV and sexual assault events. The intent was to educate police officers about the signs and symptoms of TBI and identify hurdles to use a diagnostic tool effectively and accurately on-call by police officers. Furthermore, increased TBI awareness and education may promote trust between law enforcement and victims of violent crimes with potential TBI. Additionally, "best practice" guidelines would emerge to protect victims with TBI from DV as early as the initial police encounter, and then provide acute medical assistance from a forensic nurse, and offer immediate shelter at a collaborative partner, the Sojourner Center. By consenting to the medical examination, those who have experienced strangulation or domestic violence can engage with a medical navigator and a social worker advocate, who ensures the victim can pursue medical rehabilitation not previously available.

As a progressive police agency striving to improve services to the members of the community it serves, MPD engaged with this project. While internally reviewing existing protocols and current service levels, MPD recognized the lack of training and experience to investigate cases that may involve TBI. For example, injury-induced headache, ringing in the 
ears, and language processing difficulty may be perceived by an officer as non-compliance during an interview, rather than acute symptoms of a possible TBI.

In the setting of a DV call, conventional police approaches have not emphasized the potential impact of TBI. At the outset, TBI is a complex and individual medical condition. Signs and symptoms of TBI can resemble the effects of drug and alcohol consumption, which can co-exist during DV and sexual assaults. Under the influence of drugs or alcohol, a victim (or assailant) can be difficult to question about the events of the reported incident (Evans, Schreiber Compo, \& Russano, 2009). The victim may not be impaired by drugs and alcohol, but rather their behavior, lack of clear thinking, changing story, difficulty in reading and understanding the consent forms, among other behaviors, can emerge as acute signs and symptoms of TBI (Shaw, 2002). In addition, TBI concurrent with DV does not necessarily leave visible injuries for police officers to document and report. Lastly, DV or IPV incidents rarely have additional witnesses to interview, where children in the home may be the only ones (Robinson, 2015), thereby limiting the investigations conducted by police officers. To this end, many police departments have little experience with TBI as a component of DV investigations.

The coalition clarified opportunities for the MPD to improve the quality of service to the victims of DV and sexual assault. At a minimum, officers trained to recognize signs and symptoms of TBI approach the victim differently and their additional training may result in victims receiving medical attention, evaluation, treatment and rehabilitation for TBI that they may not have previously been offered or thought necessary. Ultimately, through changes in legislation, health care professionals could testify in court that an offender's actions caused the victim to suffer a TBI. In some instances, the cycle of violence can be broken for that victim. 
One of the authors $(\mathrm{MH})$, who at the time were assigned as a sergeant for one of the Domestic Violence Units, successfully lobbied for the MPD to participate in the TBI project. After a discussion and review of existing policies, there were no contraindications to develop and implement such policy. Participation in the TBI project provided an opportunity to improve service to the citizens by individual officers and the department, as part of a broader countywide coalition.

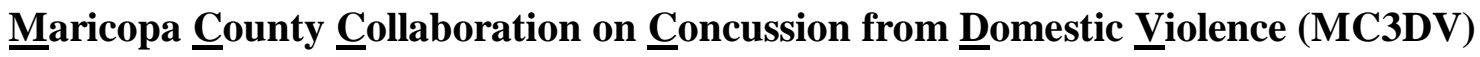

\section{Overview}

During the planning stages, the coalition of involved entities determined that the victim would need to proceed through several steps, which triaged the case from a "criminal matter" to a "healthcare matter". Through the Sojourner MC3DV entity, multiple entities interact with the victim (patient), each with a specific role. With a long-term focus on the welfare of the victim, the remainder of this article will refer to these individuals as patients, instead of domestic assault victims.

\section{First Contact - Police Officer}

The first contact with the patient is made by the police officer that responds to the initial call for police service. If the patient alleges that either a sexual assault and/or strangulation occurred while in a DV setting, the officer initiates the protocol as follows. If the scene necessitates immediate medical attention, such as consensual sexual play, autoerotic asphyxiation, and hanging, paramedics and other service providers would intervene, rather than patrol officers. The officer advises the patient that during these types of violent events, it is possible that they may have experienced a concussion. The officer asks the patient if they would complete a short screening test to assist medical personnel in the diagnosis of a concussion during the encounter. 
If the patient consents, the officer retrieves the ConQVerge device (see below), instructs the patient regarding the test, and completes the approximately two-minute test (Figure 1). The ConQVerge device is a digital ultrasonic tool with an on-board microprocessor to measure near point of convergence and accommodation, which are sensitive bioindicators of concussion (Sussman, Ho, Pendharkar, \& Ghajar, 2016). The officer records the consent and successful completion of the test. At a later time, supervisors retrieve four points of data from the ConQVerge device for researchers to compare with results obtained by the forensic nurses and neurological follow-up.

\section{Second Contact - Forensic Nurse Examiner}

After consenting to medical evaluation, the patient is transported or directed to a forensic nursing facility for a SANE. In our coalition, the SANE nurses administer sexual assault examinations, as well as forensic nursing examinations (FNE) related to strangulation. At the forensic nursing facility (Mesa Family Advocacy Center), the SANE nurse completes the appropriate examination and asks the patient for verbal consent to additional concussion testing as part of the medical examination (approved by HonorHealth Institutional Review Board). The SANE nurse provides discharge instructions to the patient, specifically written for TBI in the context of DV. Informed consent further allows contact information to be released to a patient navigator/advocate.

\section{Third Contact - Navigator/Advocate}

The patient navigator/advocate is provided with only the name and preferred contact information (e.g. text, phone, email) of the patient. No details of the suspected crime, or of the SANE/FNE exam are provided to the navigator. The navigator encourages the patient to seek further medical help based on the possibility of sustained or new neurological symptoms. Using 
a trauma-informed approach (Cronholm \& Dichter, 2018), the navigator offers to coordinate medical follow up with established concussion specialists, including transportation as needed.

\section{Implementation in the Police Department}

\section{Background}

To determine the impact on the police department to implement the additional $\mathrm{TBI} /$ concussion protocol, crime report statistics were reviewed and analyzed. In the previous calendar year (2016), approximately 160 incidents of sexual assault and strangulation involving DV were reported and investigated by MPD. Established procedures for sexual assault and strangulation assessments were already in place with county resources for SANE/FNE testing, and had been for several years. The initial investigating officer, upon learning of the allegation of the crime, sought consent from the patient to conduct the NPC with the ConQVerge device and pursue a SANE consultation. If consent was provided, the testing was completed in a Family Advocacy Center in a standardized manner at a negotiated cost per patient covered by MCAO. The protocol was normalized, accepted, and expected within the department for the officer or a detective to wait at the Family Advocacy Center with the patient, whenever practical, until the completion of the examinations. In order to incorporate TBI assessments, devices and departmental support were required.

\section{ConQVerge Device Acquisition}

Among the initial signs and symptoms of TBI is a lack of convergence of the eyes on an object near the face (NPC)(Pearce, Sufrinko, Lau, Henry, Collins, \& Kontos, 2015). Eye control and movement is a neurological function that can be measured objectively at the crime scene by a police officer using simple equipment. TBI can compromise the ability of the two eyes to work in concert to produce a single coherent image, thereby causing double vision for the patient. The 
distance at which a single object remains a single image (rather than double) is the NPC, and concussion extends this distance from $\sim 5 \mathrm{~cm}$ to $>10 \mathrm{~cm}$ (Pearce et al., 2015). To capture this information and encourage the patient to seek treatment, our coalition procured ConQVerge devices (High Tech Eye Tech LLC, Granger, IN) to measure the NPC and Near Point of Accommodation on scene by patrol officers. At the start, a minimum number of devices were distributed to patrol supervisors, who could make them available for use by patrol officers during initial contact with patients. These forty-five devices were purchased with grant funding.

\section{Departmental Support}

The use of resources and increased patrol officer time required formal support from the Chief of Police. A coalition member ( $\mathrm{MH})$ made presentations to the involved patrol Commanders and later the Executive Staff, including the legal counsel of the police department. The background, purpose, and protocols were discussed to provide a comprehensive overview of projected impact on existing resources. Further discussion covered the necessity of the program and benefit for the health of the community and individual patients.

\section{Implementation}

Once the project was formally approved and supported by the Department's Executive Staff, meetings were held with the DV detectives and their supervisors. This police department is divided into four (4) patrol divisions, each having dedicated DV detectives who report to their own supervisor. The goal was to ensure that the DV detectives could be a resource for their respective patrol divisions to address questions related to the project. Meetings with the detectives provided instruction on using the ConQVerge device as well as training to recognize signs and symptoms of TBI. 
A training video was determined to be the most efficient way to instruct the more than 330 patrol officers on how to use the ConQVerge device. A MCAO videographer produced a video tailored to the project that outlined the purpose of the project, the flow of the patient through the process, and ultimately how to use the device. The primary emphasis was on the success of the program starting with the patrol officer.

To accompany the training video, an article explaining the project with a question and answer section was distributed to all patrol squads. Each patrol squad, consisting of officers and a sergeant, meets before the start of their shift to discuss relevant daily matters. The patrol sergeants were instructed to play the video and provide answers to officers' basic questions. Officers were also instructed to contact a DV detective, if they had further questions or concerns about the project.

After the devices were distributed, patrol sergeants were given two weeks to ensure that the training was provided to all officers across the department. The officers were instructed to document in their police report if a patient consented to the NPC test, with no other information in the report. Patrol officers were to provide the ConQVerge to an investigative sergeant, who retrieved and coded the data on an internal spreadsheet. Data remain available for ongoing analysis through an honest broker mechanism.

\section{Initial Findings}

This article provides a framework for others to initiate and replicate ongoing efforts by the MPD to incorporate the lessons learned within their own established departmental structure and protocols. By gathering data from DV calls, the frequency of patients who refused any type of testing whatsoever was collected. After nine months since implementation of the protocol, MPD had 163 total reported incidents. Cases in which the patient was either a juvenile or an adult who 
was already in the hospital receiving treatment would be excluded from analysis. With the exclusion of those categories, 122 cases would qualify for the TBI protocol. Of those, 46 patients (37.7\%) refused any additional testing. Having never previously collected or analyzed these data, further investigation is needed to appreciate the challenges in providing services to our citizens.

A second observation was the patrol officers' failure to comply with the TBI portion of the protocol. Of the 122 eligible reported cases, on 44 occasions (36.1\%), the patrol officers failed to offer the patients the initial screening with the ConQVerge device. The reasons for failing to use the device on scene included an unavailability of the device and a chaotic scene. Lack of compliance to the departmental protocol prompted bi-monthly reminders to officers and sergeants about participation in the program. Additionally, the Assistant Chief of Police followed up with an email, indicating the Department's continued support of the TBI project.

\section{Lessons Learned}

The patrol sergeants recognized several factors as hurdles to implement the program. The lessons learned from first attempts to involve the police department in a system of care include (1) training, (2) patrol officer buy-in, (3) organizational structure, and (4) availability of the ConQVerge device.

\section{1) Training}

Over the first nine months of the protocol, several officers stated that they were not familiar with how to operate the ConQVerge device and were not comfortable with it. It is possible that during the training video, officers did not pay attention to the key instructions, failed to retain the information, or were not present at the time it was offered. A more formalized, structured training on the usage of the device could include a hands-on demonstration immediately following the training video. Additionally, with an average of less than 200 eligible cases in a 
year and almost 300 patrol officers, many officers may never use the device, or they may not use it for more than a year after the initial training. Having a hands-on demonstration would ensure each officer has at least used the device before being called upon to use it in a real-life scenario.

Additionally, in-person training on signs and symptoms of TBI may have increased confidence among officers' during the initial response. Having a greater understanding of TBI would likely enhance the officer's ability to communicate with the patients on that first contact, and empathetically encourage a patient to receive further evaluation and treatment, to ensure they do not suffer long term effects of TBI.

\section{2) Patrol Officer Buy-in}

By nature, law enforcement officers are a skeptical group of individuals. The job requires objective investigation and requires asking the right questions. In our efforts to initiate this project, it was believed by some officers that the project was for an individual's research paper or master's thesis. As a result, some officers did not recognize the impact of the project to provide a higher level of care to those who have experienced domestic violence. The patrol officers would be the first opportunity to navigate individuals towards medical treatment and rehabilitation, and to ultimately break the cycle of violence through facilitated patient advocacy. When officers fail to understand the purpose behind an expectation, they may fail to put forth the expected effort of compliance to the protocol. Unfortunately, projects can fall by the wayside if officers do not have buy-in. Our approach towards greater compliance included communication from administration, repeated supervisor encouragement, and clarity in presenting the vision of the countywide MC3DV project. These approaches to build patrol officer buy-in may extend to reduce any implicit bias in assigning responsibility.

\section{3) Organizational Structure}


The decentralization of DV detectives and supervisors throughout the city's jurisdiction may have contributed negatively to protocol compliance. Without a dedicated DV or special victims unit (SVU) supervised by a single supervisor, there was no single point of contact or specific, cohesive group to drive the project forward. The authors' positional authority in the MPD organizational structure lacked significant influence when the protocol was initiated. Had the information and directive first come from the Chief or Executive Committee, greater compliance could have been achieved. For the MPD, eight investigative sergeants (two at each of the four divisions) hold the same responsibilities. Of those responsibilities, DV is one part, thereby diluting the attention to this project; it is hypothesized that if the DV duties and responsibilities were centralized into one SVU, specific attention to training and compliance could be achieved.

\section{4) Availability of the ConQVerge device}

During the planning process, financial support was a limiting factor. The minimum required distribution of ConQVerge devices was to each of the 45 patrol supervisors, thereby having at least one device available in each of the four divisions, 24 hours a day. Funding was secured for 45 devices. Over the first nine months of the project, several incidents with possible TBI occurred were investigated by a patrol officer, but the sergeant was unavailable to make the device available for use. Had each, or many more officers, been assigned a device, protocol compliance at the scene may have been better.

\section{Discussion}

As of this writing, the MPD is approximately nine months into the project. The lessons learned deserve significant attention. First, how might enhanced training of officers affect the rate of compliance in using the device? Next, how might the degree of training influence the rate at which the patients consent to testing? Further consideration and evaluation is merited to 
examine reasons why victims decline medical services and testing after these highly volatile incidents, and how officers can more effectively encourage them to cooperate. The lessons learned are also limitations to be addressed in future attempts at implementation, particularly with regard to training, device usage, and case outcomes.

Police departments are encouraged to provide DV and sexual assault victims the highest quality of care and attention, beyond just the criminal investigation. Within a department, advocates and community partnerships can be called upon while the patrol officer is on scene to help provide services and resources. A police department needs to evaluate the effectiveness of existing partnerships and availability of resources, prior to implementing similar protocols and services.

To truly serve a community, as every police officer swears to do, individuals and departments must continually look for ways to improve. In this project, we believe improvement may come via research, which may identify a common link between DV and TBI. It may also improve an officer's knowledge of the effects of TBI and the traumatic stress caused during these incidents. Training provided by the program allows officers to apply that knowledge to ensure a more reliable and effective police investigation. Furthermore, it may also help develop a sense of trust by the victim towards the officer, and demonstrate the officer's true concern for that victim's well being. This trust can positively affect a victim's receptivity to available resources. Additional benefits, including rehabilitation of neurocognitive, vision, and balance deficits resulting from head blow(s), may result, with subsequent successful return to society and away from the abuser.

For the first time, we describe a process to involve police departments to identify and serve victims of domestic assault that may suffer TBI and benefit from intervention. It is anticipated 
that this report on the collaboration of law enforcement with healthcare providers, researchers, and social workers in the DV fields and the lessons learned in the conduct of this program will advance the common goal to assist victims to leave the abuser and return to society.

Acknowledgements: The authors are grateful to Maricopa County Attorney William Montgomery for leadership, insight, and support to initiate the MC3DV program. ConQVerge devices were purchased with a grant from the Hickey Family Foundation.

Declaration of Interest Statement: Hirsch Handmaker, MD is the Founder and Managing Member of Conquering Concussions, LLC. Conquering Concussions sells and distributes the ConQVerge device. Other authors have nothing to disclose with respect to the content of this manuscript. 


\section{References:}

Block, C. R., \& Devitt, C. A. (2000). The Chicago women's health risk study: report to the National Institute of Justice, June 2000 Chicago, IL: Illinois Criminal Justice Information Authority.

Block, C. R., \& Devitt, C. A. (2004). The Chicago Women's Health Risk Study at a glance Chicago, IL: Illinois Criminal Justice Information Authority.

Cronholm, P. F., \& Dichter, M. E. (2018). The Need for Systems of Care and a TraumaInformed Approach to Intimate Partner Violence. Am Fam Physician, 97(11), p Online. Retrieved from https://www.ncbi.nlm.nih.gov/pubmed/30215942

Evans, J. R., Schreiber Compo, N., \& Russano, M. B. (2009). Intoxicated witnesses and suspects: Procedures and prevalence according to law enforcement. Psychology, Public Policy, and Law, 15(3), pp. 194-221. doi:10.1037/a0016837

Glass, N., Laughon, K., Campbell, J., Block, C. R., Hanson, G., Sharps, P. W., \& Taliaferro, E. (2008). Non-fatal strangulation is an important risk factor for homicide of women. Journal of Emergency Medicine, 35(3), pp. 329-335. doi:10.1016/j.jemermed.2007.02.065

Hawley, D. A., McClane, G. E., \& Strack, G. B. (2001). A review of 300 attempted strangulation cases Part III: injuries in fatal cases. Journal of Emergency Medicine, 21(3), pp. 317-322.

McClane, G. E., Strack, G. B., \& Hawley, D. (2001). A review of 300 attempted strangulation cases Part II: clinical evaluation of the surviving victim. Journal of Emergency Medicine, 21(3), pp. 311-315.

Pearce, K. L., Sufrinko, A., Lau, B. C., Henry, L., Collins, M. W., \& Kontos, A. P. (2015). Near Point of Convergence After a Sport-Related Concussion: Measurement Reliability and Relationship to Neurocognitive Impairment and Symptoms. American Journal of Sports Medicine, 43(12), pp. 3055-3061. doi:10.1177/0363546515606430

Robinson, J. (2015). The experience of the child witness: Legal and psychological issues. Int J Law Psychiatry, 42-43, pp. 168-176. doi:10.1016/j.ijlp.2015.08.022 Retrieved from https://www.ncbi.nlm.nih.gov/pubmed/26343312

Shaw, N. A. (2002). The neurophysiology of concussion. Progress in Neurobiology, 67(4), pp. 281-344.

Strack, G. B., McClane, G. E., \& Hawley, D. (2001). A review of 300 attempted strangulation cases. Part I: criminal legal issues. Journal of Emergency Medicine, 21(3), pp. 303-309.

Sussman, E. S., Ho, A. L., Pendharkar, A. V., \& Ghajar, J. (2016). Clinical evaluation of concussion: the evolving role of oculomotor assessments. Neurosurgery Focus, 40(4), p E7. doi:10.3171/2016.1.focus 15610 


\section{Figure Captions:}

Figure 1. Victim and patient navigation through the Maricopa County Collaboration on Concussion in Domestic Violence protocol.

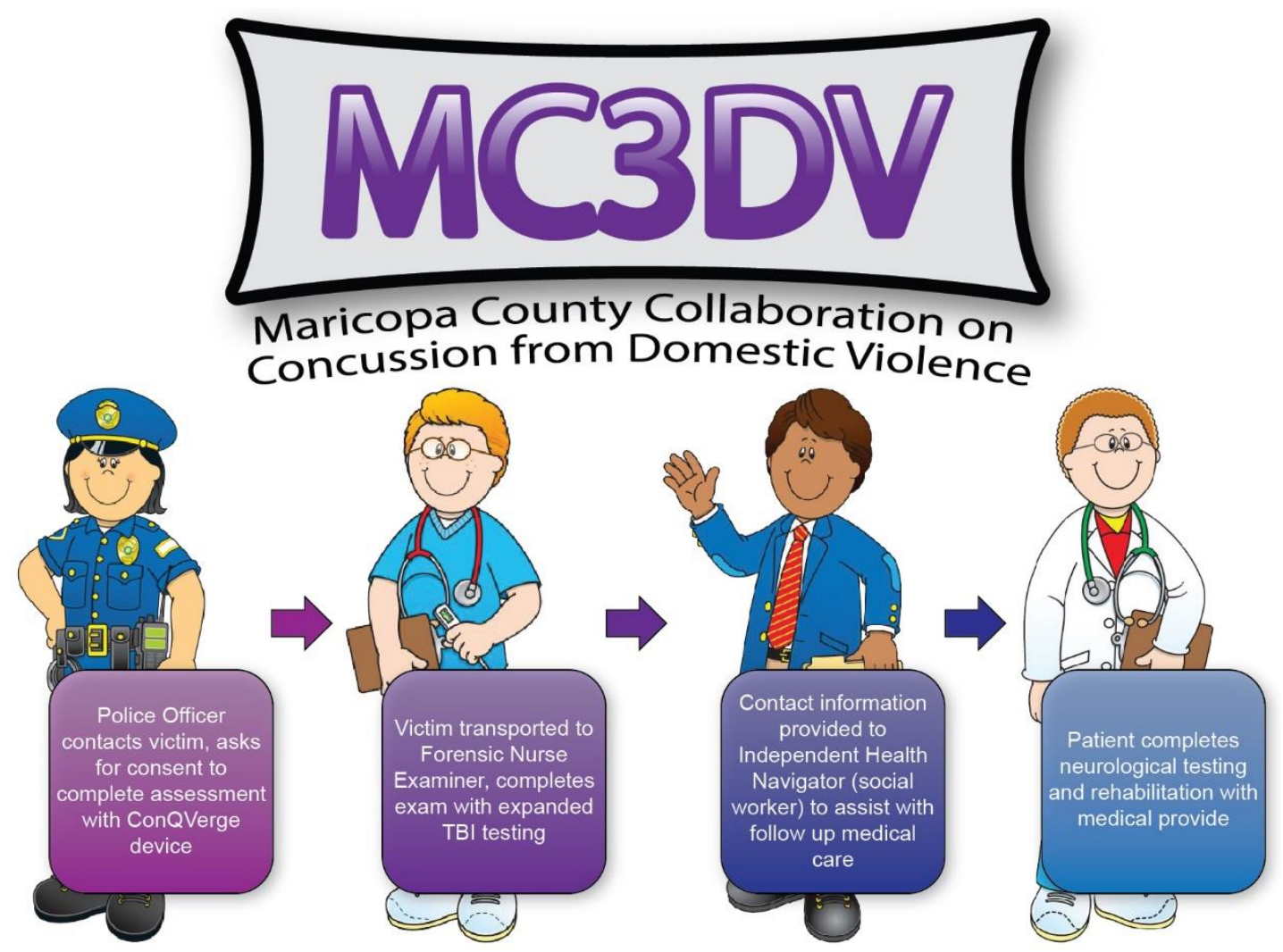

Figure 2. Lieutenant Mark Higbee demonstrating the use of the ConQVerge near point convergence device on a subject.

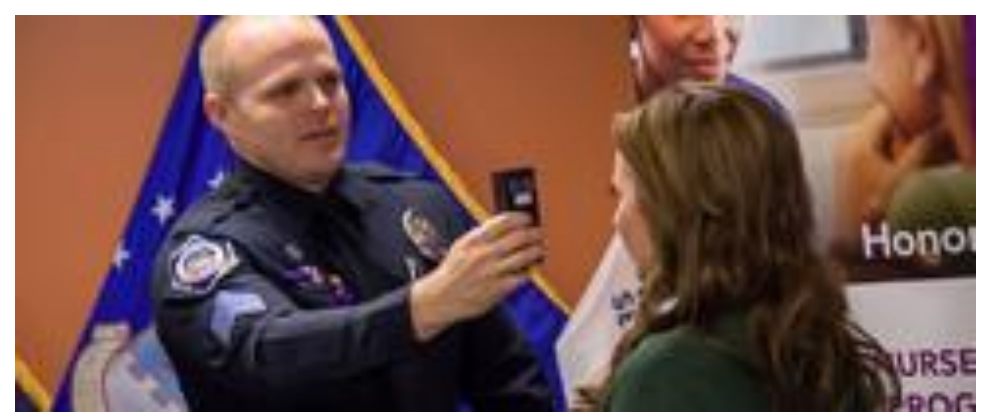

